\title{
Deep insights hidden in beautiful metaphors - remembering E. C. G. Sudarshan
}

\author{
Anil Shaji* \\ School of Physics, IISER Thiruvananthapuram, Vithura 695 551, India
}

The most valuable lesson that I learned from my association with E. C. G. Sudarshan was on the sheer joy of scientific discovery. The joy of figuring things out. This was not a lesson that he ever talked about. On the contrary, he would try to impress upon all his students that theoretical physics is $99 \%$ donkey work as he would put it. As if it was just back-breaking tedium with little reward. Peeping around the kitchen wall into the living room late at night at his modest house in Austin, Texas, USA, I used to see him the way I like to remember him. Sitting on the recliner, the TV on and running probably a tele-shopping channel, notepad in hand, calculating away.

I was a PhD student then and those were the days when I would give him company at his place when his wife was travelling. Sudarshan may even be working on a problem that the two of us would have discussed earlier in the day at his office. Typically the professor would expect the graduate student to attack the problem and find a way forward and report about it the next day. Here the professor was happily working away on it while, the Ph D student was scrounging for snacks in the kitchen. When the solution to the problem came, Sudarshan would become all animated and excited. Just like a little boy getting hold of a new toy. He would explain the whole thing to me then and there even if, as it happened sometimes, he was working on a problem that was part of some other Ph D student's work. Half awake at 2 a.m., I rarely understood the solution or the explanation, but his joy and excitement were contagious and without speaking about it he showed me the rewards that lay at the end of the $99 \%$ donkey work.

I was fortunate to be able to work on a wide variety of topics ranging from open quantum dynamics to the spin statistics connection during my $\mathrm{PhD}$ and I realize now that it was possible only because in Sudarshan I had a thesis adviser who could give me able guidance in pretty much any area of physics because he himself had made seminal and original contributions to most of those areas. Associating with him and discussing on all these topics, I got a glimpse of the depth and range of his genius and insight. In building the Standard Model of particle physics, none other than Steven Weinberg has stated categorically that the $\mathrm{V}-\mathrm{A}$ theory of weak interactions developed

*e-mail: shaji@iisertvm.ac.in by Sudarshan and Marshak and later elaborated upon by Gellmann and Feynman was key to developing the theory of electro weak interactions for which Glashow, Salam and Weinberg received the Nobel Prize in 1979. The Universal Four-Fermi interaction proposed by Sudarshan was without question an addition of the most fundamental nature to our understanding of the workings of the physical universe. Sudarshan gave me an old copy of the Padua conference proceedings paper in which he and Marshak had proposed the Universal Four-Fermi interaction, and what I found most impressive was as a graduate student just as I was then - Sudarshan had written in no uncertain terms that four experiments had to be re-done since they contradicted his formulation of the theory. Indeed they were re-done and proven to be incorrect while simultaneously proving Sudarshan right. Around the time I was reading the paper, the Physical Review was digitizing all their archives and Feynman and Gellmann's paper on the same topic was available in digital form while Sudarshan's Padua Conference paper was not. I felt this was unfair and proceeded to type up the paper once again in LaTeX from the hardcopy that I had. I wished to put the digitized version on the arXiv pre-print server but Sudarshan felt that would not be appropriate.

Sudarshan's way of describing the electromagnetic field (light) quantum mechanically was without question the correct one, and it also made a clear connection with the well-developed theory of traditional classical optics. The first paper developing the mathematics required to describe the non-ideal case of real-world quantum particles that lie in contact with their surroundings was by Matthews, Rau and Sudarshan in 1961. A seminal paper on an important sub-field of the same topic, namely continuous descriptions of open dynamics was by Kossakowski, Gorini and Sudarshan. Now the field is of great relevance in quantum computing, quantum information and quantum technologies. The quantum Zeno effect was a theoretical prediction by Sudarshan and Misra that captured the imagination of many people due to its philosophical implications. However, the real beauty of it is that the effect could be demonstrated in the lab and now it is something that is actively pursued so as to reach the goal of controlling individual atoms and other quantum systems. Sudarshan also laid the foundations of the field theory of particles that travel at speeds strictly greater than the speed of light - tachyons as we know them now. 
Indeed there are many other areas of theoretical physics in which the unassuming physicist from Kerala had contributed greatly, including foundations of quantum mechanics, statistical physics, etc.

There has been quite a bit of discussion, especially in India, about whether Sudarshan was denied a much deserved Nobel Prize. In my opinion there is no question that he deserved the Prize. In fact, he should have been given due recognition for each of the seminal works mentioned earlier. Maybe that was the problem too. The Prize seems to favour individuals who made a single seminal contribution and then continued to work in the same field making incremental contributions to it for the rest of their careers. So maybe one can speculate that the Prize never came his way because here was a man who made Nobelworthy contributions in not just one but many fields; something perhaps way beyond what a single prize committee can fully appreciate. If the Nobel Prize were like the Oscars instead, recognizing the important contributions of the previous year, maybe Sudarshan may have had five of them on his mantlepiece!

Ultimately the reward was the joy of discovery. Disappointment at the lack of deserved recognitions was limited in his characteristic humility to sarcastic remarks punctuating his lectures like 'I wrote down this particular equation first and so it is now known by somebody else's name!' He was also willing to ask difficult questions even in public regarding exactly who did what and when in the course of scientific discovery. Did Einstein appropriate S. N. Bose's result on what is now called the BoseEinstein statistics, or did Einstein genuinely promote the discovery and add substance to it? Einstein's first wife Mileva's name was on the initial draft of his 1905 paper on the special theory of relativity. Why was it not there in the published version? Such questions indirectly belied the fact that Sudarshan did harbour a sense of being cheated out of due credit at several occasions for work he had done.

Sudarshan had a deep and commanding voice, particularly when he was talking about physics. It was perhaps a bit intimidating too. But even now, when I am stuck with a problem, not able to find a way forward, I long to hear it again. He would not probably know the exact answer to the problem, but his insight into the working of the physical universe was so deep that what he would say in such situations would be typically a cryptic analogy or even an anecdote related to village life in verdant Kerala, where I also come from. If you think about it deeply enough, it would reveal his insight into the problem and it would lead you to the solution.

doi: $10.18520 / \mathrm{cs} / \mathrm{v} 116 / \mathrm{i} 2 / 227-228$ 